\title{
An acoustic mechanism for core-collapse supernova explosions
}

\author{
A. Burrows ${ }^{a, *}$, E. Livne $^{\mathrm{b}}$, L. Dessart ${ }^{\mathrm{c}}$, C.D. Ott ${ }^{\mathrm{d}}$, J. Murphy $^{\mathrm{e}}$ \\ a Department of Astronomy, University of Arizona, Tucson, AZ 85721, USA \\ ${ }^{\mathrm{b}}$ Racah Institute of Physics, The Hebrew University, Jerusalem, Israel \\ ${ }^{\mathrm{c}}$ Department of Astronomy, University of Arizona, Tucson, AZ 85721, USA \\ ${ }^{\mathrm{d}}$ Max-Planck-Institut für Gravitationsphysik, Albert-Einstein-Institut, Golm/Potsdam, Germany

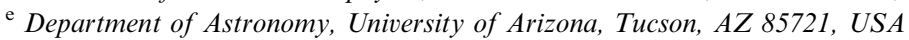

Available online 24 July 2006

\begin{abstract}
We present a new mechanism for core-collapse supernova explosions that relies upon acoustic power generated in the inner core as the driver. In our simulation using an 11-solar-mass progenitor, a strong advective-acoustic oscillation à la Foglizzo with a period of 25$30 \mathrm{~ms}$ arises $200 \mathrm{~ms}$ after bounce. Its growth saturates due to the generation of secondary shocks, and kinks in the resulting shock structure funnel and regulate subsequent accretion onto the inner core. However, this instability is not the primary agent of explosion. Rather, it is the acoustic power generated in the inner turbulent region and most importantly by the excitation and sonic damping of core g-mode oscillations. An $l=1$ mode with a period of $3 \mathrm{~ms}$ grows to be prominent around $500 \mathrm{~ms}$ after bounce. The accreting protoneutron star is a self-excited oscillator. The associated acoustic power seen in our 11-solar-mass simulation is sufficient to drive the explosion. The angular distribution of the emitted sound is fundamentally aspherical. The sound pulses radiated from the core steepen into shock waves that merge as they propagate into the outer mantle and deposit their energy and momentum with high efficiency. The core oscillation acts like a transducer to convert accretion energy into sound. An advantage of the acoustic mechanism is that acoustic power does not abate until accretion subsides, so that it is available as long as it may be needed to explode the star.
\end{abstract}

(c) 2006 Elsevier B.V. All rights reserved.

PACS: 97.60.Bw; 96.60.Ly; 97.60.Jd; 26.30.+k; 25.30.-c; 26.50.+x

Keywords: Supernovae: general; Neutrinos; Stellar oscillations; Nucleosynthesis

\section{Contents}

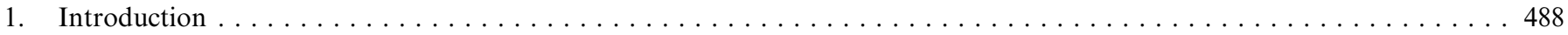

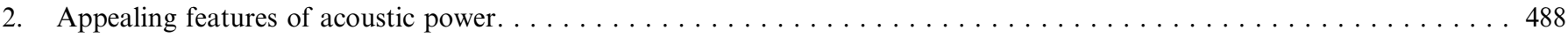

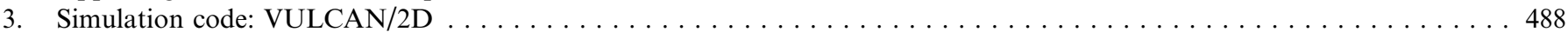

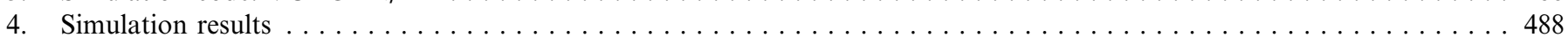

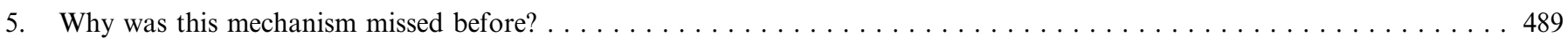

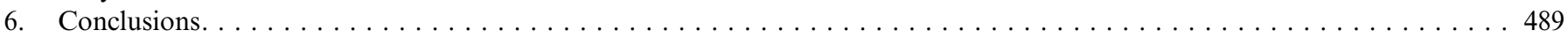

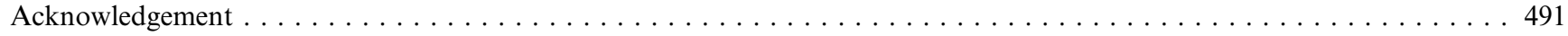

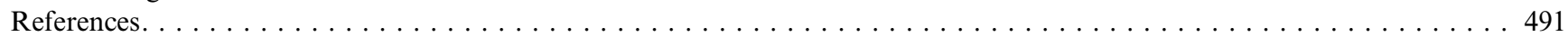

\footnotetext{
* Corresponding author.

E-mail address: aburrows@as.arizona.edu (A. Burrows).
} 


\section{Introduction}

The essence of the mechanism of core-collapse supernovae must be the conversion of a fraction of the reservoir of gravitational energy into the kinetic and internal energy of the exploding mantle of the Chandrasekhar core whose instability inaugurates core collapse. However, despite decades of work on the direct hydrodynamic and the neutrino mechanisms, both in 1D and multi-D, these mechanisms have yet to be shown to lead robustly to explosion for a healthy range of progenitor masses and the best physics. If the energy transfer from the core to the mantle necessary to explode the star is by neither direct hydrodynamics nor neutrino heating, what is left? How do core-collapse supernovae explode? Burrows et al. (2006) propose a new alternative, the generation in the core and the propagation into the mantle of strong sound waves. Acoustic power is, potentially, an efficient means to transport energy and momentum into the outer mantle to drive the supernova explosion. Unlike neutrinos, sound is almost $100 \%$ absorbed in the matter. As sound pulses propagate outward down the density gradient they steepen into multiple shock waves that catch up to one another and merge. If sufficient sound is generated in the core, it would be a natural vehicle for the gravitational energy of infall to be transferred to the outer mantle and could be the key missing ingredient in the core-collapse explosion mechanism. Furthermore, periodic shocking due to multiple sound pulses can lead naturally to entropies in the debris of hundreds of units, just what is required for r-process nucleosynthesis (Woosley and Hoffman, 1992; Woosley et al., 1994; Hoffman et al., 1996).

Through our recent $2 \mathrm{D}$ radiation/hydrodynamic simulations, we have identified a vigorous source for the necessary acoustic power: the excitation and oscillation of core pulsation modes in the deep interior of the PNS. We have discovered that turbulence and anisotropic accretion in the inner $40-100 \mathrm{~km}$ can excite and maintain vigorous core $\mathrm{g}$ mode oscillations which decay by the radiation of sound. The inner core acts as a transducer for the conversion of accretion gravitational energy into acoustic power. The associated acoustic power seen in our simulations is sufficient to drive the explosion $>550 \mathrm{~ms}$ after bounce.

\section{Appealing features of acoustic power}

There are certain virtues to acoustic driving that bear mentioning. First is that while the acoustic luminosity is much smaller than the neutrino luminosity, almost all of the sound is absorbed in the mantle matter. At late times in our simulation, less than a percent of the $v_{\mathrm{e}}$ and $\bar{v}_{\mathrm{e}}$ neutrino luminosity is absorbed. This amounts to an neutrino absorption power of $\leqslant 10^{50} \mathrm{erg} \mathrm{s}^{-1}$, compared with an estimated core acoustic power at the end of our calculation near $\sim 10^{51} \mathrm{erg} \mathrm{s}^{-1}$.

Second, sound carries not only energy, but momentum, and this factor seems to be important in our simulations.
The momentum flux for sound with the same energy flux as neutrino radiation is larger by the ratio of the speed of light to the speed of sound, which in the inner mantle regions is as much as a factor of ten. Third, acoustic power propagates from where it is generated to where it is needed; it fulfills the central requirement of a core-collapse supernova mechanism that it involve energy transfer from the bound interior PNS to the outer exploding mantle. If the acoustic power is large enough, it is the ideal transfer agent. Fourth, the acoustic source seems to grow just when the neutrino luminosity is ebbing and, importantly, it continues until explosion ensues. Fifth, the successive merger of trains of sound waves that steepen into shocks provides a non-neutrino way to entropize some of the matter and naturally achieve r-process conditions.

\section{Simulation code: VULCAN/2D}

We have used the code VULCAN/2D for our 2D supernova simulations. VULCAN/2D uses the hydrodynamic approach described in Livne (1993), with the transport methods discussed in Livne et al. (2004) and Walder et al. (2005). The Multi-Group, Flux-Limited Diffusion (MGFLD) variant is a Newtonian, 2D, multi-group radiation/hydrodynamics code with an Arbitrary-LagrangianEulerian (ALE) structure (with remap). Velocity terms in the transport sector, such as Doppler shifts, are not included in the code, though advection is. The flux limiter is a vector version of the one found in Bruenn (1985). The code can handle rotation. In $2 \mathrm{D}$, the calculations are axially/azimuthally symmetric, and we use cylindrical coordinates ( $r$ and $z$ ), but the grid points themselves can be placed at arbitrary positions. This allows us to employ a Cartesian grid at the center (inner $\sim 20 \mathrm{~km}$ ) and transition to a spherical grid further out. The grid resolution is essentially uniform everywhere within $\sim 20 \mathrm{~km}$. A version of this grid structure is plotted in Ott et al. (2004). The Cartesian format in the interior allows us to avoid the severe Courant problems encountered in 2D by all other groups employing grid-based codes due to the inner angular Courant limit and, thereby, to perform the calculations in full $2 \mathrm{D}$ all the way to the center.

\section{Simulation results}

Burrows et al. (2006) have recently focussed on the 11$M_{\odot}$ progenitor without rotation of Woosley and Weaver (1995). The calculations were done from $\sim 200 \mathrm{~ms}$ before bounce to $\sim 1.0 \mathrm{~s}$ after bounce, significantly longer than any other previous multi-D simulations.

At $\sim 50 \mathrm{~ms}$ after bounce the shock has stalled, is roughly spherical, and is at a spherical radius $(R)$ of $\sim 115 \mathrm{~km}$. Neutrino-driven convection has begun in the region $\sim 50 \mathrm{~km}$ wide interior to the shock wave. By $\sim 150 \mathrm{~ms}$, the average shock radius has reached $\sim 150 \mathrm{~km}$, and the convection is encompassing the region down to $R \sim 75 \mathrm{~km}$. In the full angular region of $180^{\circ}$, we see 5-6 dominant turbules 
(eddies) with angular scales of $\sim 30^{\circ}-35^{\circ}$. Near $\sim 200 \mathrm{~ms}$, the average shock radius has receded back to $\sim 110 \mathrm{~km}$. If shock recession had been our criterion for failure, we might have stopped the calculation here. However, at around $\sim 200 \mathrm{~ms}$, the shock is beginning to wobble up and down perceptibly in an $\ell=1$ mode with a period near $25-30 \mathrm{~ms}$ and a $\Delta R / R$ near $25 \%$. At $\sim 250 \mathrm{~ms}$, the $\Delta R / R$ is approaching $\sim 50 \%$ and the up-down asymmetry is quite pronounced. The growth time for the shock anisotropy varies, but is near $50-100 \mathrm{~ms}$. We identify this early quasi-periodic oscillation with the advective-acoustic instability and the "standing accretion shock instability" (SASI) suggested by Foglizzo (2001a,b), Foglizzo and Tagger (2000), Foglizzo et al. (2005), and by Blondin et al. (2003). By $\sim 300-350 \mathrm{~ms}, \Delta R / R$ has grown to a factor of two and the wobble is taking on a more vigorous character. In fact, this vigor results in the emergence of a secondary shock wave; the flow now has nested shock waves. The creation of secondary shocks serves to saturate the amplitude of the shock oscillation, which nevertheless continues. Importantly, at the shock intersection kinks, the accretion through the outer shock is channeled and penetrates in lower entropy streams into the interior and onto the core.

By $500 \mathrm{~ms}$ after bounce, the acoustic power from the core is quite pronounced and is starting to power outflow and the beginnings of an explosion. After $\sim 500 \mathrm{~ms}$, and certainly by $550 \mathrm{~ms}$, the sonic power, though its early source was the turbulence around the core region, is driven mostly by a core oscillation that is being excited by the violent accretion streams. This oscillation can be seen as early as $\sim 350 \mathrm{~ms}$ after bounce and has a period near $\sim 3 \mathrm{~ms}$, very much smaller than the sound-travel-time in the shocked cavity. It is predominantly an $\ell=1 \mathrm{~g}$-mode of the inner $\sim 40 \mathrm{~km}$ that has grown strong over a period of $\sim 100 \mathrm{~ms}$ to reach nonlinear amplitudes by $\sim 500 \mathrm{~ms}$. This mode would have been suppressed had we excised the inner core, not performed the calculations over the full $180^{\circ}$, or performed the simulations in $1 \mathrm{D}$ interior to some convenient radius.

The core oscillation is driven by the energy in the accretion streams and by the turbulence around the core, both of which ultimately derive their energy from the gravitational energy of infall. The oscillation is damped by sound waves that emerge out of the core. These sound waves steepen into shock waves, and, by dint of their momentum and energy flux, "ignite" the supernova explosion. The core oscillation is acting like a transducer for the conversion of the gravitational energy of infall into radiating acoustic power and at the later stages is a far more important source of acoustic power than the inner turbulence. Moreover, from $\sim 400 \mathrm{~ms}$ to $\sim 660 \mathrm{~ms}$ the efficiency for the conversion of accretion power into sound power is increasing. As long as the accretion continues during this phase, the core oscillation seems to be driven and the sound is emitted. After the explosion has progressed sufficiently and accretion subsides, the core oscillation decays and the sound source abates. It seems that as long as the acoustic power due to core oscillation is needed to drive the explosion, it is available. If the neutrino mechanism does not abort this scenario by inaugurating an earlier explosion, this may be a natural self-regulating mechanism for the supernova phenomenon.

\section{Why was this mechanism missed before?}

It is important to list the reasons the acoustic phenomena we have identified and presented were not seen before. First, most calculations were stopped after the shock radius first subsided around 200-300 ms after bounce, but before the shock instability was much in evidence, and before turbulence around the core could generate significant acoustic power. Second, those calculations that were not stopped early were continued because they experienced an early neutrino-driven, multi-D explosion. Such an explosion arose either naturally from the particular code being used, or was artificially produced. If the explosion commences early, the PNS core oscillations are not excited to useful amplitudes and the shock instability is more mild. Third, and most importantly, to date all other grid-based supernova codes have conducted calculations either with the cores excised, handled in $1 \mathrm{D}$, or on a $\sim 90^{\circ}$ wedge, thereby completely suppressing core oscillations and the resulting $\ell=1$ acoustic flux. Such procedures can even muffle the acoustic flux generated in the turbulent inner "convective" zones. As a result of some combination of the reasons above, no previous supernova simulations, before those using VULCAN/2D, could have discovered the acoustic driving and core oscillation mechanism. One key to the discovery of this potentially important mechanism was the computational liberation of the inner core to execute its natural multi-dimensional motions. Another key was patience to perform the simulations to very late times.

\section{Conclusions}

We have discovered a new mechanism for core-collapse supernova explosions that focusses on the acoustic power generated in the core region. The strength, radiation pattern, and character of the emergent sound are influenced by the shock instability that arises after $\sim 200 \mathrm{~ms}$, but this instability is not the agent of explosion. Rather, it is the acoustic power generated first in the turbulent region around the inner PNS core and then through the excitation and sonic damping of core g-mode oscillations. An $\ell=1$ mode grows at late times to be prominent around $\sim 500 \mathrm{~ms}$ after bounce, though it is in evidence as early as $\sim 300 \mathrm{~ms}$ after bounce. In our Newtonian simulation, its period is $\sim 3 \mathrm{~ms}$. At the end of the calculation, this core g-mode contains $\sim 10^{50} \mathrm{ergs}$, is radiating sound into the exploding mantle at a rate near $\sim 10^{51} \mathrm{erg} \mathrm{s}^{-1}$, and has a Q-value of $\sim 200$. At late times, but before explosion, the accreting protoneutron star is a self-excited oscillator. After the acoustic power becomes strong, the average direction of the radiated sound and the angular positions 
of the exciting plumes outside the core are anti-correlated. Since core $\ell=0$ modes are not excited to an appreciable degree, the driving acoustic radiation pattern is fundamentally anisotropic and the initial phase of the explosion is unipolar. Fig. 1 depicts an entropy map versus time for the directions along the positive and negative poles, and clearly portrays the pulsations, the onset of explosion, the high entropies possible, and the long time delay. Due to the extreme breaking of spherical symmetry, this model manifests simultaneous accretion and explosion.

An advantage of the acoustic mechanism is that acoustic power does not abate until accretion subsides, so that it is available as long as it is needed. This is not the case for neutrinos, whose luminosities and mantle heating rates inexorably decrease at late times. Hence, this may be the long-sought-after self-regulating mechanism of the supernova energy and, being a function of the accretion rate, is determined mainly by the progenitor density structure, with some ambiguity due to the sensitivity to initial conditions in chaotic flows and due to rotation. Furthermore, unlike neutrinos, sound pulses that steepen into shock waves are almost completely absorbed in the baryonic outer mantle. Moreover, sound deposits not only energy, but momentum. Ironically, the large accretion rates that were thought to inhibit explosion in the neutrino-driven mechanism are not a disadvantage in the acoustic model. In fact, the very accretion that might be inhibiting the neutrino mechanism facilitates the acoustic mechanism, but at later times. Furthermore, by their nature the accretion funnels constitute a disproportionate share of the infalling material. The result is that while at the same time these streams are exciting the core oscillations accretion from the other directions is weaker, thereby presenting less of an obstacle in those directions to eventual explosion. In our calculation with the $11-M_{\odot}$ progenitor, the delay to explosion is $\sim 550 \mathrm{~ms}$. This is longer than the delay tradi-

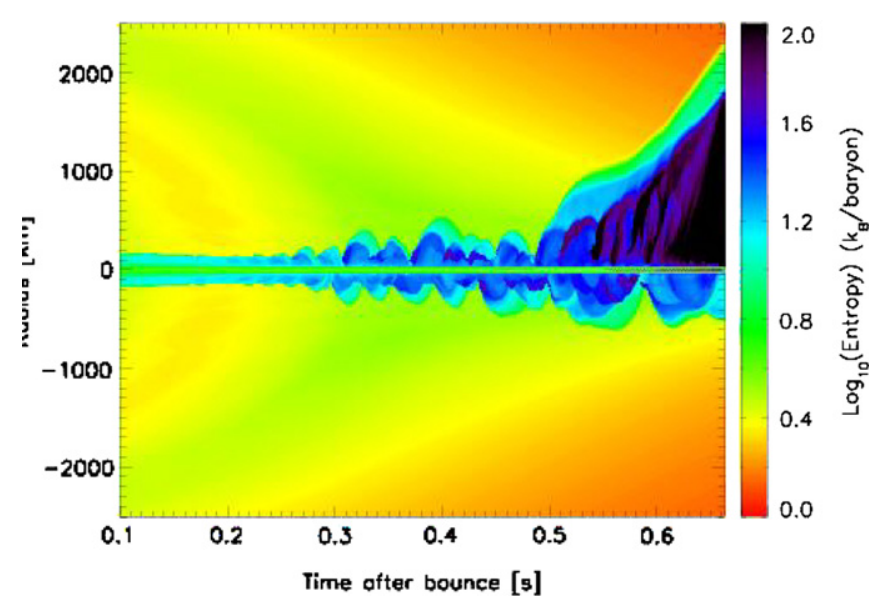

Fig. 1. Time evolution, from $100 \mathrm{~ms}$ to $\sim 660 \mathrm{~ms}$ after core bounce, of the entropy (logarithmic scale) along the axis of symmetry, i.e., at $r=0$ or latitudes $\pm 90^{\circ}$, and covering the inner $2500 \mathrm{~km}$ of the grid. An entropy ceiling of 100 has been adopted. tionally associated with the onset of multi-D neutrino-driven explosions ( 200-300 ms).

The baryon mass of our remnant neutron star is $\sim 1.42 M_{\odot}$, with a gravitational mass near $\sim 1.3 M_{\odot}$. This is close to what is canonically expected from measured values. Had the core exploded much earlier, the mass remaining might have been uncomfortably lower.

Though we find that an $\ell=1$ g-mode eventually dominates, $\ell=2$ and $\ell=3$ modes and harmonics are in evidence and there is also likely to be nonlinear mode-mode coupling. Unlike $\ell=1$ modes, $\ell=2$ modes will generate gravitational radiation and will do so at a characteristic frequency (!) that is a function of the EOS, relativity, and the PNS structure. Fig. 2 shows a frequency-time plot of the core pulsations for the first second after bounce. The various modes (and their temporal evolution!) jump out cleanly. In the excitation of normal modes in the supernova context, we may have a direct signature of core physics and supernova phenomenology.

It may be that better neutrino and weak-interaction physics, full 3D radiation/hydrodynamic simulations, multi-group/multi-angle simulations, a new suite of progenitor models, the use of other massive-star progenitors (we have here studied only one), or some qualitatively important flaw in our approach or implementation will alter our conclusions here. The role of rotation must be explored, for both the neutrino and the acoustic models. The multi-D neutrino-driven mechanism may still obtain, particularly for the lowest mass progenitors. The papers by Janka et al. $(2005 a, b)$ contain the suggestion that the neutrino mechanism might still obtain for the lowest mass

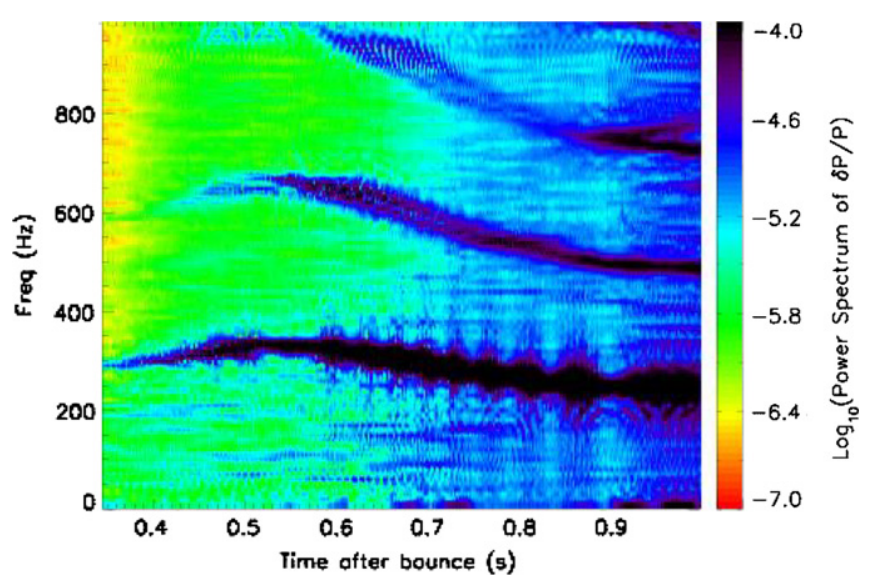

Fig. 2. Colorscale of the power spectrum of the fractional pressure variation $\left(P(R, \theta)-\langle P(R, \theta)\rangle_{\theta}\right) /\langle P(R, \theta)\rangle_{\theta}$ at a radius $R=30 \mathrm{~km}$, as a function of time after bounce and frequency. For each time $t$, a power spectrum is calculated from a sample of time snapshots covering $t \pm 50 \mathrm{~ms}$, at a resolution of $0.5 \mathrm{~ms}$. Note the emergence of power in the $\sim 330 \mathrm{~Hz}$ ( $\equiv 3 \mathrm{~ms}$ ) g-mode, as well as the strengthening $\ell=2$ harmonic mode near $\sim 675 \mathrm{~Hz}$ at late times. The latter is of relevance for gravitational radiation emission. Note also the late-time evolution of the characteristic frequencies. Such evolution can be quite instructive. (For interpretation of the references to color in this figure legend, the reader is referred to the web version of this article.) 
massive stars when the full $180^{\circ}$ computational domain (even without liberating the core to execute oscillations) and state-of-the-art Boltzmann transport are incorporated. These authors seem to see the onset of explosion for an 11.2- $M_{\odot}$ model from Woosley et al. (2002). Interestingly, this model has a density cliff and a much steeper mantle density gradient around $1000-2000 \mathrm{~km}$ than the $11-M_{\odot}$ model from Woosley and Weaver (1995) on which we focussed in Burrows et al. (2006). However, the acoustic mechanism is the only one identified to date that may work as well or better for more massive progenitors, that produces entropies necessary for the r-process, that leaves behind neutron-star masses in the observed range, that produces blast top-bottom asymmetries similar to those seen in SN1987A, and that has the potential to simultaneously explain pulsar kicks by simple core recoil (Burrows et al. 2006, in preparation). Time (and further calculation) will reveal whether the new acoustic mechanism, with all its exciting implications for core-collapse explosions, actually obtains in Nature.

\section{Acknowledgement}

We acknowledge support for this work from the Scientific Discovery through Advanced Computing (SciDAC) program of the DOE, grant number DE-FC0201ER41184, and from the NSF under grant AST0504947. E.L. thanks the Israel Science Foundation for support under grant \# 805/04, and C.D.O. thanks the Albert-Einstein-Institut for providing CPU time on their Peyote Linux cluster. This research used resources of the National Energy Research Scientific Computing Center, which is supported by the Office of Science of the U.S. Department of Energy under Contract No. DE-AC0376SF00098.

\section{References}

Blondin, J.M., Mezzacappa, A., DeMarino, C., 2003. ApJ 584, 971. Bruenn, S.W., 1985. ApJS 58, 771.

Burrows, A., Livne, E., Dessart, L., Ott, C.D., Murphy, J., 2006. ApJ 640, 878.

Foglizzo, T., 2001a. AstAp 368, 311.

Foglizzo, T., 2001b. AstAp 392, 353.

Foglizzo, T., Tagger, M., 2000. AstAp 363, 174.

Foglizzo, T., Galletti, P., Ruffert, M., 2005. AstAp 435, 397.

Hoffman, R.D., Woosley, S.E., Fuller, G.M., Meyer, B.S., 1996. ApJ 460, 478

Janka, H.-T., Buras, R., Kifonidis, K., Marek, A., Rampp, M., 2005. Cosmic Explosions, On the 10th Anniversary of SN1993J. In: Marcaide, J.M., Weiler, Kurt W. (Eds.), Proceedings of IAU Colloquium 192. Springer Proceedings in Physics, vol. 99. Springer, Berlin, p. 253 (astro-ph/0401461).

Janka, H.-Th., Buras, R., Kitaura Joyanes, F.S., Marek, A., Rampp, M., Scheck, L., 2005. Neutrino-driven supernovae: An accretion instability in a nuclear physics controlled environment. In: Proceedings of the 8th International Symposium on Nuclei in the Cosmos, Vancouver, Canada, July 19-23, 2005. Nuclear Physics A, 758, 19-26.

Livne, E., 1993. ApJ 412, 634.

Livne, E., Burrows, A., Walder, R., Thompson, T.A., Lichtenstadt, I., 2004. ApJ 609, 277.

Ott, C.D., Burrows, A., Livne, E., Walder, R., 2004. ApJ 600, 834.

Walder, R., Burrows, A., Ott, C.D., Livne, E., Lichtenstadt, I., Jarrah, M., 2005. ApJ 626, 317.

Woosley, S.E., Hoffman, R.D., 1992. ApJ 395, 202.

Woosley, S.E., Wilson, J.R., Mathews, G.J., Hoffman, R.D., Meyer, B.S., 1994. ApJ 433, 229.

Woosley, S.E., Weaver, T.A., 1995. ApJS 101, 181.

Woosley, S.E., Heger, A., Weaver, T.A., 2002. RvMP 74, 1015. 